\title{
Radiation Oncology reviewer acknowledgement 2014
}

Claus Belka ${ }^{1}$ and Thalyana Smith-Vikos ${ }^{2^{*}}$

\section{Contributing reviewers}

The editorial team of Radiation Oncology would like to thank all our reviewers who have contributed to the journal in Volume 9 (2014).

\author{
Yasser Abo-Madyan \\ Germany
}

Sebastian Adamczyk

Poland

Dario Agnese

Italy

Merina Ahmed

United Kingdom

Mario Airoldi

Italy

Alireza Akhondi-Asl

United States of America

Markus Alber

Denmark

Francesca Albertini

Switzerland

Valerie Albrecht

Germany

Manuel Algara

Spain

Filippo Alongi

Italy

Mohamad Al-Sheikhly

United States of America

Natasa Anastasov

Germany
Nicolaus Andratschke

Switzerland

C Nicolaj Andreassen

Denmark

Mekhail Anwar

United States of America

Meritxell Arenas

Spain

Dirk Arnold

Germany

Vasileios Askoxylakis

Germany

Myriam Ayadi

France

Omid Azimzadeh

Germany

Woo Kyun Bae

South Korea

Amit Bahl

India

Kurt Baier

Germany

Rinske Bakker

Netherlands

Hendrik Ballhausen

Germany

\author{
Dimos Baltas \\ Germany \\ James Balter \\ United States of America \\ Robyn Banerjee \\ Canada \\ Mark Bangert \\ Germany \\ Philip Bardin \\ Australia
}

Zarko Barjaktarovic

Germany

Chris Barker

United States of America

Guido Baroni

Italy

Tristan Barrett

United Kingdom

Michael Barton

Australia

Brigitta Baumert

Germany

Beth Beadle

United States of America

Sergio Benavente

Spain

* Correspondence: radiationoncology@biomedcentral.com

BioMed Central, Floor 6, 236 Gray's Inn Road, London WC1X 8HB, UK

1 Department of Radiation Oncology, University Hospital of Munich,

Marchioninistr. 15, 81377, Munich, Germany 
Bernhard Berger

Germany

Eric Berthelet

Canada

Dirk Beutner

Germany

Albert Biete

Spain

Mario Bignardi

Italy

Tina Binderup

Denmark

Wolfgang Birkfellner

Austria

Marc Bischof

Germany

Pierre Blanchard

France

Oliver Blanck

Germany

Sanne Blinde

Netherlands

Andreas Block

Germany

Felix Böckelmann

Germany

Judit Boda-Heggemann

Germany

Stefan Boeck

Germany

Edwin Boelke

Germany

Beat Bojaxhiu

Switzerland

Fabrice Bonnet

France

Alp Özgün Börcek

Turkey

Tom Boterberg

Belgium

Dirk Bottke

Germany

Ugo Bottoni

Italy
Michael Brada

United Kingdom

Julie Bradley

United States of America

Herbert Braselmann

Germany

Michael Bremer

Germany

Giuseppe Brisinda

Italy

James Brown

United Kingdom

Paul Brown

United States of America

Gregor Bruggmoser

Germany

Thomas Brunner

Germany

Vicente Bruzzaniti

Italy

Wilfried Budach

Germany

Krzysztof Bujko

Poland

Ralph Bundschuh

Germany

Jan Bussink

Netherlands

Karl Butterworth

United Kingdom

Felipe Calvo

Spain

Mauricio Campos Daziano

Chile

Aras Emre Canda

Turkey

Lynnette Cary

United States of America

Richard Castillo

United States of America

Annemieke Cats

Netherlands

Charles Catton

Canada
Jimmy Caudell

United States of America

Jean-Michel Caudrelier

Canada

Guillaume Cazoulat

France

Laura Cella

Italy

Francesco Cellini

Italy

Laura Cerezo

Spain

Anuradha Chakravarthy

United States of America

Marc Chamberlain

United States of America

Annie Chan

United States of America

Albert Chang

United States of America

Joe Chang

United States of America

Ming-Chih Chang

Taiwan

Samuel Chao

United States of America

Naved Chaudhri

Germany

\section{Buxin Chen}

United States of America

Delphine Chen

United States of America

Ming Chen

China

Ronald Chen

United States of America

Ui-Kyu Chi

South Korea

Kwan-Hwa Chi

Taiwan

Kevin Choe

United States of America

Hans Christiansen

Germany 
Daniel Chua

Hong Kong

Michael Chuong

United States of America

Nikola Cihoric

Switzerland

Savino Cilla

Italy

Mario Ciocca

Italy

Alessandro Clivio

Switzerland

Anthony Cmelak

United States of America

Sean Collins

United States of America

Rob Coppes

Netherlands

Gennaro Cormio

Italy

Luca Cozzi

Italy

Gilles Crehange

France

Hans Crezee

Netherlands

M.A. Cuesta

Netherlands

Johan Peter Cuijpers

Netherlands

Fred Currell

United Kingdom

Alan Dal Pra

Switzerland

Megan Daly

United States of America

Sidsel Damkjaer

Denmark

Rolando M. D'Angelillo

Italy

Indra Das

United States of America

Prajnan Das

United States of America
Gert De Meerleer

Belgium

Antonella De Rosa

Italy

Armando De Virgilio

Italy

letizia deantonio

Italy

Joseph Deasy

United States of America

Melanie Deberne

France

Robert Den

United States of America

Carmen Dence

United States of America

Timm Denecke

Germany

Francesco Deodato

Italy

Beatrice Detti

Italy

Andreas Dinkel

Germany

Oliver Dohm

Germany

Jacobson Don

United States of America

Qinghua Dong

China

Dimitrios Dougenis

Greece

Stephen Dowdell

Australia

Weiliang Du

United States of America

Yadranko Ducic

United States of America

Marciana Nona Duma

Germany

Peter Dunscombe

Canada

Fréderic Duprez

Belgium
Marco Durante

Germany

Raghav Dwivedi

United Kingdom

Yvonne Dzierma

Germany

Thomas Eade

Australia

Michael Eble

Germany

Franziska Eckert

Germany

Monika Ecsedy

Hungary

Jens Edmund

Denmark

Avraham Eisbruch

United States of America

Hoda Elbakry

Egypt

John Eley

United States of America

Olgun Elicin

Switzerland

Lorraine Elit

Canada

Michael Elliott

United States of America

Nagy Elsayyad

United States of America

Medhat ElSebaie

Egypt

SR Emani

India

Svend Engelholm

Denmark

Benedikt Engels

Belgium

Mustafa Esassolak

Turkey

Marion Essers

Netherlands

Veni Ezhil

United Kingdom 
Khashayar Fakhrian
Germany

Aurora Fassi

Italy

Dimitrios Fatouros

Greece

Barry Feig

United States of America

Steven Feigenberg

United States of America

Pascal Fenoglietto

France

Claudio Festuccia

Italy

Christian Fiandra

Italy

Kathryn Field

Australia

Rainer Fietkau

Germany

Andrea Riccardo Filippi

Italy

Jacob Finkelstein

United States of America

Michele Fiore

Italy

Alba Fiorentino
Italy

Michael K Fix

Switzerland

Jochen Fleckenstein

Germany

Gerald Fogarty

Australia

Antonella Fogliata

Switzerland

Emmanouil Fokas

United Kingdom

Nicolas Foray

France

Kenneth Forster

United States of America

Piero Fossati

Italy
Eugene Fourkal

United States of America

Claudia Fournier

Germany

Giovanni Franchin

Italy

Nicolaas Franken

Netherlands

Thomas Friedrich

Germany

Junichi Fukada

Japan

Simone Fulda

Germany

Christoph Fürweger

Germany

Nobukazu Fuwa

Japan

Udo Gaipl

Germany

Razvan Galalae

Germany

Maria Antonietta Gambacorta Italy

Robin Garcia

France

Elisabetta Gargioni

Germany

Cristina Garibaldi

Italy

Laurie Gaspar

United States of America

Harriet Gee

Australia

Ian Geh

United Kingdom

Dietmar Georg

Austria

Tawfik Giaddui

United States of America

Chiara Gianoli

Italy

Iris Gibbs

United States of America
David Gierga

United States of America

Suki Gill

Australia

Frank Giordano

Germany

Meredith Giuliani

Canada

Christoph Glanzmann

Switzerland

Bengt Glimelius

Sweden

Rob Glynne-Jones

United Kingdom

Ziya Gokaslan

United States of America

Daniel Gomez

United States of America

Sara González

Argentina

Holger Gottschlag

Germany

Julie Goudreault

Canada

Gerhard Grabenbauer

Germany

Christian Graeff

Germany

Joel Greenberger

United States of America

Christopher Gregory

United Kingdom

Daila Gridley

United States of America

Perry Grigsby

United States of America

Anca Grosu

Germany

Peter Gruebling

Germany

Tejpal Gupta

India

Sarah Gwynne

United Kingdom 
Jonathan Haas

United States of America

Gregor Habl

Germany

Scott Hadley

United States of America

Matthias Häfner

Germany

Christopher Hallemeier

United States of America

Gerry Hanna

United Kingdom

Gerard G Hanna

United Kingdom

Wendy Hara

United States of America

Nicholas Hardcastle

Australia

Josefin Hartmann

Germany

Christina Haston

Canada

Birgitte Havelund

Denmark

Maria Hawkins

United Kingdom

Xia He

China

Felix Heinemann

Germany

Christian Heinz

Germany

Frank Hensley

Germany

Klaus Herfarth

Germany

Dwight Heron

United States of America

Julia Heß

Germany

Ekkehard Hewer

Switzerland

Reinhard Heyd

Germany
Tarek Hijal

Canada

Robin Hill

Australia

Daisaku Hirano

Japan

Ying Hitchcock

United States of America

Ivy Ho

Singapore

James Hodge

United States of America

Frank Hoebers

Netherlands

Fatemeh Homaeishandiz

Iran

Ulrich Theodor Hopt

Germany

Orla Howe

Ireland

Morten Høyer

Denmark

Feng-Ming Hsu

Taiwan

Chaosu Hu

China

Weigang $\mathrm{Hu}$

China

Maarten CCM Hulshof

Netherlands

Dezheng Huo

United States of America

Sefik Igdem

Turkey

George Iliakis

Germany

Hiroshi Inoue

Japan

Edy Ippolito

Italy

Hassan Iqbal

Pakistan

Hiromichi Ishiyama

Japan
Fumiaki Isohashi

Japan

Jun Itami

Japan

Seong Soon Jang

South Korea

Marek Janiak

Poland

Nathalie Jansen

Germany

Maha Jawad

United States of America

Supriya Jayant Sastri

India

Urszula Jelen

Germany

Verena Jendrossek

Germany

Alexandra D. Jensen

Germany

J. Martin Jensen

Canada

Jae-Uk Jeong

South Korea

Branislav Jeremic

Serbia

Xun Jia

United States of America

Esther Jimenez-Jimenez Spain

Hosang Jin

United States of America

Meredith Johnston

Australia

Peter Johnstone

United States of America

Nuria Jornet

Spain

Mirjana Josipovic

Denmark

German Juan

Spain

Alain Jung

France 
Markus Juonala

Finland

Johannes Kaanders

Netherlands

Josephine Kang

United States of America

Tom Karagiannis

Australia

Giorgos Karakousis

United States of America

Sana Karam

United States of America

Christian P. Karger

Germany

Sonja Katayama

Germany

Hiroyuki Katoh

Japan

David Kaul

Germany

Chul-Seung Kay

South Korea

Ludwig Keilholz

Germany

Monika Keller

Germany

Markus Kellermeier

Germany

Lucyna Kepka

Poland

Ki Chang Keum

South Korea

YongKan Ki

South Korea

Merrill Kies

United States of America

David Kim

Canada

Grace Kim

United States of America

Jihun Kim

United States of America

Jin Sung Kim

South Korea
Jaehyup Kim

United States of America

Kwang Seok Kim

South Korea

Mi-Sook Kim

South Korea

Dae Yong Kim

South Korea

Su Ssan Kim

South Korea

Yongbok Kim

United States of America

Young Seok Kim

South Korea

Christian Kirisits

United Kingdom

Stephan Kloeck

Switzerland

Sebastian Klüter

Germany

Andrew Kneebone

Australia

Lukas Knybel

Czech Republic

Thomas Koch

Germany

Stefan Koerber

Germany

Kiyotaka Kohshi

Japan

Masahiko Koizumi

Japan

Marisa Kollmeier

United States of America

Bridget Koontz

United States of America

Gyoergy Kovacs

Germany

Josef Kovarik

United Kingdom

Takuyo Kozuka

Japan

Michael Kraemer

Germany
Mechthild Krause

Germany

Andra Krauze

United States of America

Robert Krempien

Germany

Marco Krengli

Italy

David Krug

Germany

Dmitri Krysko

Belgium

Andrzej Kukielka

Poland

Shaleen Kumar

India

Sanath Kumar

United States of America

Pavel Kundrat

Germany

Charles Kunos

United States of America

Guntram Kunz

Germany

Patrick Kupelian

United States of America

Abraham Kuten

Israel

Young Kwok

United States of America

Frank Lagerwaard

Netherlands

Tamara Lah

Slovenia

Vincent Lai

Hong Kong

Rachelle Lanciano

United States of America

Stephanie Lang

Switzerland

Normand Laperriere

Canada

Pedro C. Lara

Spain 
George Laramore

United States of America

Ingmar Lax

Sweden

Cathy Lazarus

United States of America

Anne Lee

Hong Kong

John Lee

Belgium

Yun-Sil Lee

South Korea

Sebastian Lettmaier

Germany

Rolf Lewensohn

Sweden

Dadong Li

United States of America

Jianbin Li

China

Taoran Li

United States of America

Zuofeng Li

United States of America

Xiaoying Liang

United States of America

Charles Limoli

United States of America

Jin-Ching Lin

Taiwan

Katja Lindel

Germany

Bodo Lippitz

Sweden

An Liu

United States of America

Chun-feng Liu

China

Meng-Zhong Liu

China

Chen Lixin

China

Simon Lo

United States of America
Luiz Lobato

United States of America

Kristina Loessl

Switzerland

Frank Lohr

Germany

Jiade Lu

Singapore

Shun Lu

China

John Lukens

United States of America

Martin Lundsgaard Hansen

Denmark

LD Lunsford

United States of America

L. Dade Lunsford MD

United States of America

Yulia Lyatskaya

United States of America

Victor Macias

Spain

Katsuya Maebayashi

Japan

Yoshihiko Maehara

Japan

Kevin Maggio

United States of America

Dennis Mah

United States of America

Karsten Mahnke

Germany

Cornelius Maihöfer

Germany

Philippe Maingon

France

Bettina Maiwald

Germany

Raymond Mak

United States of America

Chiyoko Makita

Japan

Eirik Malinen

Norway
Pietro Mancosu

Italy

Robert Mandic

Germany

Robert Maráz

Hungary

Carmelo Marino

Italy

Simone Marnitz

Germany

Michal Masarík

Czech Republic

Laura Masucci

Canada

Koji Matsuo

United States of America

Yukinori Matsuo

Japan

Christiane Matuschek

Germany

Riccardo Maurizi Enrici Italy

Andrew McDonald

United States of America

Stephen McMahon

United Kingdom

James Melotek

United States of America

Veerle Melotte

Netherlands

Cynthia Menard

Canada

Alejandra Mendez Romero

Netherlands

Oliver Micke

Germany

Gerald Mickisch

Germany

Ben Mijnheer

Netherlands

Michael Milano

United States of America

Giuseppe Minniti

Italy 
Raymond Miralbell

Switzerland

Norio Mitsuhashi

Japan

Masahiko Miura

Japan

Giulio Modorati

Italy

Raphaël Moeckli

Switzerland

Simone Moertl

Germany

Majid Mohiuddin

United States of America

Alessio G. Morganti

Italy

Masahiro Morimoto

Japan

W. James Morris

Canada

Gerard Morton

Canada

Lutz Moser

Germany

Andre Mouton

United Kingdom

Ralph Muecke

Germany

Klaus Mueller

Germany

Arndt-Christian Müller

Germany

Per Munck af Rosenschöld

Denmark

Yuji Murakami

Japan

Martin Murphy

United States of America

Lars Murrer

Netherlands

Olaf Nairz

Austria

Mitsuhiro Nakamura

Japan
Takashi Nakano

Japan

Sapna Nangia

India

Patrick Naumann

Germany

Pierina Navarria

Italy

Rodolfo Negri

Italy

Ursula Nestle

Germany

Nicole Nesvacil

Austria

Andrea Ng

United States of America

R. Charles Nichols

United States of America

Carsten Nieder

Norway

Gabriele Niedermann

Germany

Peter Niehoff

Germany

Yuzuru Niibe

Japan

Holly Ning

United States of America

Paige Nitsch

United States of America

Jenny Nobes

United Kingdom

Gianmauro Numico

Italy

Joost Nuyttens

Netherlands

Jan Nyman

Sweden

Uwe Oeh

Germany

Toshiyuki Ogata

Japan

Shinichi Ohta

Japan
Eric Ojerholm

United States of America

Didem Colpan Oksuz

United Kingdom

Murat Okutan

Turkey

Kenneth Olivier

United States of America

Ivo Olivotto

Canada

Cem Onal

Turkey

Michael Orth

Germany

Feras Oskan

Germany

Piet Ost

Belgium

Christian Ostheimer

Germany

Mattia Falchetto Osti

Italy

Oliver Ott

Germany

Natsuo Oya

Japan

Mahmut Ozsahin

Switzerland

Chiara Paganelli

Italy

David Palma

Canada

Joshua Palmer

United States of America

Jianji Pan

China

Valerie Panet-Raymond

Canada

Alexandros Papachristofilou

Switzerland

Evangelos Pappas

Greece

Dillip Kumar Parida

India 
François Paris

France

Paolo Passoni

Italy

Abhijit Patel

United States of America

Arnold Paulino

United States of America

Jacob Pe'er

Israel

Cheng Peng

United States of America

Dolly Penn

United States of America

Vincent Pennaneach

France

J.J. Penninkhof

Netherlands

Joseph Pepek

United States of America

Rodrigo Perez

Brazil

Francesco Perri

Italy

Nadeem Pervez

Canada

Gianfranco Pesce

Switzerland

Maria Grazia Petrongari Italy

Heike Peulen

Netherlands

Asja Pfaffenberger

Germany

Thomas Pfluger

Germany

Daniel Pham

Australia

Justin Phillips

United States of America

Alessia Pica

Switzerland

Steffi Ulrike Pigorsch

Germany
Karsten Pilones

United States of America

Nuno Pimentel

Portugal

Michael Pinkawa

Germany

Tomasz Piotrowski

Poland

Ludwig Plasswilm

Switzerland

Julio Plata Bello

Spain

George Plataniotis

United Kingdom

Volker Platz

Germany

Alan Pockley

United Kingdom

Wojciech Polkowski

Poland

Bruce Pollock

United States of America

Bjoern Poppe

Germany

Vincent Potiron

France

Per Rugaard Poulsen

Denmark

Bas Pouw

Netherlands

Olivier Pradier

France

Heru Prasetio

Germany

Brendan Prendergast

United States of America

Kevin Prise

United Kingdom

Martin Pruschy

Switzerland

Newell Pugh

United States of America

Paul Martin Putora

Switzerland
Florian Putz

Germany

Hongryull Pyo

South Korea

Ibrahim Qaddoumi

United States of America

Hai Qu

United States of America

Harvey Quon

Canada

Dirk Rades

Germany

Ganesh Radhakrishna

United Kingdom

Zvi Ram

Israel

Giuliano Ramadori

Germany

Sara Ramella

Italy

Ulla Ramm

Germany

Ganesh Rao

United States of America

Coen Rasch

Netherlands

Andrew Rauth

Canada

Michael Reardon

United States of America

Anne Hansen Ree

Norway

Valerie Reed

United States of America

Marc Remke

Canada

James Renaud

Canada

Swaroop Revannasiddaiah India

Nadeem Riaz

United States of America

Marco Riboldi

Italy 


\begin{tabular}{|c|c|c|}
\hline $\begin{array}{l}\text { Umberto Ricardi } \\
\text { Italy }\end{array}$ & $\begin{array}{l}\text { Elvio Grazioso Russi } \\
\text { Italy }\end{array}$ & $\begin{array}{l}\text { Manfred Schmidt } \\
\text { Germany }\end{array}$ \\
\hline $\begin{array}{l}\text { Andreas Richter } \\
\text { Germany }\end{array}$ & $\begin{array}{l}\text { Jean-claude Rwigema } \\
\text { United States of America }\end{array}$ & $\begin{array}{l}\text { Uwe Schneider } \\
\text { Switzerland }\end{array}$ \\
\hline $\begin{array}{l}\text { Christian Richter } \\
\text { Germany }\end{array}$ & $\begin{array}{l}\text { Mohammadsaeed Saboori } \\
\text { Germany }\end{array}$ & $\begin{array}{l}\text { Max Schnurr } \\
\text { Germany }\end{array}$ \\
\hline $\begin{array}{l}\text { Daniel Richter } \\
\text { Germany }\end{array}$ & $\begin{array}{l}\text { Sotaro Sadahiro } \\
\text { Japan }\end{array}$ & $\begin{array}{l}\text { Michael Scholz } \\
\text { Germany }\end{array}$ \\
\hline $\begin{array}{l}\text { Jens Ricke } \\
\text { Germany }\end{array}$ & $\begin{array}{l}\text { Azmat Sadozye } \\
\text { United Kingdom }\end{array}$ & $\begin{array}{l}\text { David Schreiber } \\
\text { United States of America }\end{array}$ \\
\hline $\begin{array}{l}\text { Ron Riesenburger } \\
\text { United States of America }\end{array}$ & $\begin{array}{l}\text { Arjun Sahgal } \\
\text { Canada }\end{array}$ & $\begin{array}{l}\text { Ulrich Schüller } \\
\text { Germany }\end{array}$ \\
\hline $\begin{array}{l}\text { Oliver Riesterer } \\
\text { Switzerland }\end{array}$ & $\begin{array}{l}\text { Masatoshi Saito } \\
\text { Japan }\end{array}$ & $\begin{array}{l}\text { Amanda Schwint } \\
\text { Argentina }\end{array}$ \\
\hline $\begin{array}{l}\text { Andreas Rimner } \\
\text { United States of America }\end{array}$ & $\begin{array}{l}\text { Ladan Saleh-Ebrahimi } \\
\text { Germany }\end{array}$ & $\begin{array}{l}\text { Marta Scorsetti } \\
\text { Italy }\end{array}$ \\
\hline $\begin{array}{l}\text { Mark Rivard } \\
\text { United States of America }\end{array}$ & $\begin{array}{l}\text { Riccardo Santoni } \\
\text { Italy }\end{array}$ & $\begin{array}{l}\text { Clemens Seidel } \\
\text { Germany }\end{array}$ \\
\hline $\begin{array}{l}\text { Mack Roach } \\
\text { United States of America }\end{array}$ & $\begin{array}{l}\text { Joseph Santoro } \\
\text { United States of America }\end{array}$ & $\begin{array}{l}\text { Robert Semrau } \\
\text { Germany }\end{array}$ \\
\hline $\begin{array}{l}\text { David Roberge } \\
\text { Canada }\end{array}$ & $\begin{array}{l}\text { Naoko Sanuki-Fujimoto } \\
\text { Japan }\end{array}$ & $\begin{array}{l}\text { Mehmet Sen } \\
\text { United Kingdom }\end{array}$ \\
\hline $\begin{array}{l}\text { Stephen Roberts } \\
\text { United Kingdom }\end{array}$ & $\begin{array}{l}\text { Yoko Satoh } \\
\text { Japan }\end{array}$ & $\begin{array}{l}\text { Matteo Seregni } \\
\text { Italy }\end{array}$ \\
\hline $\begin{array}{l}\text { Franz Rödel } \\
\text { Germany }\end{array}$ & $\begin{array}{l}\text { William Sause } \\
\text { United States of America }\end{array}$ & $\begin{array}{l}\text { Marco Serpa } \\
\text { Germany }\end{array}$ \\
\hline $\begin{array}{l}\text { George Rodrigues } \\
\text { Canada }\end{array}$ & $\begin{array}{l}\text { Bryan Schaly } \\
\text { Canada }\end{array}$ & $\begin{array}{l}\text { Waseem Sharieff } \\
\text { Canada }\end{array}$ \\
\hline $\begin{array}{l}\text { Erik Roelofs } \\
\text { Netherlands }\end{array}$ & $\begin{array}{l}\text { Daniel Schanne } \\
\text { United States of America }\end{array}$ & $\begin{array}{l}\text { Gregory Sharp } \\
\text { United States of America }\end{array}$ \\
\hline $\begin{array}{l}\text { Marianne Roenjom } \\
\text { Denmark }\end{array}$ & $\begin{array}{l}\text { Daniel Schanne } \\
\text { United States of America }\end{array}$ & $\begin{array}{l}\text { Yuta Shibamoto } \\
\text { Japan }\end{array}$ \\
\hline $\begin{array}{l}\text { Dirk Roggenbuck } \\
\text { Germany }\end{array}$ & $\begin{array}{l}\text { Stephan Scheidegger } \\
\text { Switzerland }\end{array}$ & $\begin{array}{l}\text { Keiko Shibuya } \\
\text { Japan }\end{array}$ \\
\hline $\begin{array}{l}\text { Teboh Roland } \\
\text { United States of America }\end{array}$ & $\begin{array}{l}\text { Heike Scheithauer } \\
\text { Germany }\end{array}$ & $\begin{array}{l}\text { Helen Shih } \\
\text { United States of America }\end{array}$ \\
\hline $\begin{array}{l}\text { Olivier Rouvière } \\
\text { France }\end{array}$ & $\begin{array}{l}\text { Hans Schieferr } \\
\text { Switzerland }\end{array}$ & $\begin{array}{l}\text { Kyung Hwan Shin } \\
\text { South Korea }\end{array}$ \\
\hline $\begin{array}{l}\text { Peter Rubin } \\
\text { United States of America }\end{array}$ & $\begin{array}{l}\text { Alexander Schlaefer } \\
\text { Germany }\end{array}$ & $\begin{array}{l}\text { Yoshiyuki Shioyama } \\
\text { Japan }\end{array}$ \\
\hline $\begin{array}{l}\text { Carmen Rubio } \\
\text { Spain }\end{array}$ & $\begin{array}{l}\text { Michel Schlienger } \\
\text { France }\end{array}$ & $\begin{array}{l}\text { Ori Shokek } \\
\text { United States of America }\end{array}$ \\
\hline $\begin{array}{l}\text { Tatjana Rundek } \\
\text { United States of America }\end{array}$ & $\begin{array}{l}\text { Maximilian Schmid } \\
\text { Austria }\end{array}$ & $\begin{array}{l}\text { David Shultz } \\
\text { United States of America }\end{array}$ \\
\hline
\end{tabular}


Frank-André Siebert

Germany

Nanna Sijtsema

Netherlands

Charles Simone

United States of America

Anurag Singh

United States of America

Ben Slotman

Netherlands

Wendy Smith

Canada

Gregory Smyth

United Kingdom

Toshinori Soejima

Japan

Riccardo Soffietti

Italy

Shiyu Song

United States of America

Akinaga Sonoda

Japan

Yukihiko Sonoda

Japan

Ute Spahn

Germany

Hanno M. Specht

Germany

Lena Specht

Denmark

Michael Spiotto

United States of America

Daniel Spratt

United States of America

Michael Stahl

Germany

Lukas Stalpers

Netherlands

Roel Steenbakkers

Netherlands

Diana Steinmann

Germany

Kevin Stephans

United States of America
Florian Stieler

Germany

Alison Stillie

United Kingdom

Sandro Stoeckli

Switzerland

Igor Stojkovski

Macedonia

Markus Stoll

Germany

Bjoern Stork

Germany

Guy Storme

Belgium

Tino Streller

Switzerland

Lidia Strigari

Italy

Vratislav Strnad

Germany

Carmen Stromberger

Germany

Gabriela Studer

Switzerland

Martin Stuschke

Germany

Shinji Sugahara

Japan

Xiaonan Sun

China

Katarina Surlan Popovic

Slovenia

Clare Suttie

Australia

Phillip Taddei

Lebanon

Takeo Takahashi

Japan

Wataru Takahashi

Japan

Tetsuo Takehara

Japan

Ozgur Tanriverdi

Turkey
Mohmad Ashraf Teli

India

Chris Terhaard

Netherlands

Wolfgang Thasler

Germany

David Thomas

United States of America

CR Thomas

United States of America

Daniela Thorwarth

Germany

Zhen Tian

United States of America

Carmen Timke

Germany

Ingeborg Tinhofer

Germany

Marcello Tiseo

Italy

Florent Tixier

France

Manuel Todorovic

Germany

Yuji Toiyama

Japan

Koichi Tokuuye

Japan

Vincenzo Tombolini

Italy

Erkan Topkan

Turkey

Dunyaporn Trachootham

Thailand

Alison Tree

United Kingdom

Silke Tribius

Germany

Theresa Trotter

Canada

Pauline Truong

Canada

Erik Tryggestad

United States of America 
James Tucker

United States of America

Sandra Turner

Australia

Matthias Uhl

Germany

Kristian Unger

Germany

Dmytro Unukovych

Sweden

\section{Maurizio Valeriani}

Italy

Nicholas Van As

United Kingdom

Tara Van de Water

Netherlands

Wouter Van Elmpt

Netherlands

Dirk Van Gestel

Belgium

Paul Van Houtte

Belgium

Hilde Van Parijs

Belgium

Joep Van Roermund

Netherlands

John Randolf van Sornsen de Koste

Netherlands

Jef Vandemeulebroucke

Belgium

Hansjoerg Vees

Switzerland

Vaneja Velenik

Slovenia

Karen Venables

United Kingdom

Wilko Verbakel

Netherlands

Dirk Verellen

Belgium

Frank Verhaegen

Netherlands

Marcel Verheij

Netherlands
Daniel Vetterli

Switzerland

Gregory Videtic

United States of America

Asgaut Viste

Norway

Dirk Vordermark

Germany

Wan Zamaniah Wan Ishak

Malaysia

Hesheng Wang

United States of America

Ping Wang

China

Po-Ming Wang

Taiwan

Luhua Wang

China

Fredrik Wärnberg

Sweden

Ronald Warnick

United States of America

Samantha Warren

United Kingdom

Yoichi Watanabe

United States of America

Damien Charles Weber

Switzerland

Christian Weiss

Germany

Stefan Welz

Germany

Thomas G. Wendt

Germany

Rene Werner

Germany

John Werning

United States of America

Charlotte Westbury

United Kingdom

Greg Wheeler

Australia

Joachim Widder

Netherlands
Tilo Wiezorek

Germany

Theo Wiggers

Netherlands

Bas Wijnhoven

Netherlands

Christopher Willett

United States of America

Christopher Willey

United States of America

Lucy Wills

United Kingdom

Joerg Wischhusen

Germany

Barbara Witulla

Germany

Ulrich Wolf

Germany

Jens Wölfelschneider

Germany

Hendrik Andreas Wolff

Germany

Binbin Wu

United States of America

Q. Jackie Wu

United States of America

Jun-xin Wu

China

Qiuwen Wu

United States of America

Abraham Wu

United States of America

Florian Wuerschmidt

Germany

Evan Wuthrick

United States of America

Zhong-jun Xia

China

Lei Xing

United States of America

Ting Xu

United States of America

Yoshiya Yamada

United States of America 
Jack Yang

United States of America

Kamil Yenice

United States of America

Kheng-Wei Yeoh

Singapore

Ugur YILMAZ

France

Yong Yin

China

Hongmei Ying

China

Indra Yohannes

Germany

Young Do Yoo

South Korea

John Yordy

United States of America

Ken Yoshida

Japan
Cedric Yu

United States of America

James Yu

United States of America

Ning Yue

United States of America

Nikolaos Zamboglou

Germany

Tomas Zaremba

Denmark

Paul Zarogoulidis

Greece

Xin Zhang

United States of America

Zhen Zhang

China

Weiling Zhao

United States of America

Chong Zhao

China
Dandan Zheng

United States of America

Jinyuan Zhou

United States of America

Li Zhou

China

Hong Zhou

China

Thomas Zilli

Switzerland

Zvonimir Zore

Croatia

Zachary Zumsteg

United States of America

Brigitte Zurl

Austria

Daniel Zwahlen

Switzerland

Felix Zwicker

Germany 